\title{
AS ALTERAÇÕES CLIMÁTICAS NAS POLÍTICAS PÚBLICAS AGRÍCOLAS E DE DESENVOLVIMENTO RURAL, NO ENSINO E NA INVESTIGAÇÃO EM PORTUGAL
}

\author{
Margarida Quintela MARTINS \\ CICS.NOVA / INIAV.IP \\ Margaridaqm@gmail.com
}

\begin{abstract}
Resumo
As alterações climáticas são identificadas "como uma das maiores ameaças ambientais, sociais e económicas que o planeta e a humanidade enfrentam". Nas últimas décadas tem-se vindo a verificar um aumento considerável de secas, de cheias e outros eventos extremos. A ocorrência destes fenómenos é cada vez mais frequente e a sua intensidade também tem vindo a aumentar.

Estes e outros eventos climáticos têm vindo a acontecer à escala global e estão a afectar o equilíbrio de alguns ecossistemas e até a sua própria continuidade. Neste sentido, o tema Alterações Climáticas tem suscitado a atenção de cientistas de várias especialidades que tentam procurar soluções de adaptação e mitigação das suas consequências.

Pretende-se estudar a integração desta problemática na investigação, formação de recursos humanos e políticas públicas nas áreas da agricultura, floresta e desenvolvimento rural. Tentando perceber a evolução, continuidade e inovação, nomeadamente, no desenho das políticas propriamente ditas, na disseminação do conhecimento académico e da formação profissional dos recursos humanos ligados ao sector primário e por outro lado, perceber como tem sido o caminho traçado no âmbito do conhecimento científico ao nível das instituições de investigação, desenvolvimento e inovação. O estudo focará o Instituto Nacional de Investigação Agrária e Veterinária, I.P., Laboratório do Estado do Ministério da Agricultura, Florestas e Desenvolvimento Rural.
\end{abstract}

Palavras-chave: Agricultura e desenvolvimento rural, alterações climáticas, investigação e inovação, políticas públicas

\begin{abstract}
Climate change is identified as "one of the greatest environmental, social and economic threats facing the planet and humanity". In the last decades has been observed a considerable increase in droughts, floods and other extreme events. The occurrence of these phenomena is increasingly frequent and their intensities are also increasing.

These events occur on a global scale and are affecting the balance and even the continuity of life of various ecosystems. Climate Change is one of the main global challenges that has been studied from different perspectives with the aim of finding adaptation and mitigation solutions.

This project intends to study the integration of this problem in research, human resources training and public policies in the areas of agriculture, forestry and rural development. Trying to understand the evolution, continuity and innovation, particularly in the design of public policies, the dissemination of academic knowledge and training of human resources linked to the primary sector. On the other hand, realize how has been the path traced in the knowledge research, development and innovation institutions. The study will focus the National Institute of Agrarian and Veterinary Research, I.P., which is the State Laboratory of the Ministry of Agriculture, Forestry and Rural Development.
\end{abstract}

Keywords: Agriculture and rural development, climate change, research and innovation, public policy 


\section{Introdução}

A generalidade dos cenários relativamente a alterações climáticas de acordo com 0 Intergovernmental Panel on Climate Change - IPCC, e também apresentados por estudos elaborados no âmbito dos projectos SIAM I (2002) ${ }^{1}$, SIAM II (2006) e CLIMAAT $(2006)^{2}$, projecta para o período compreendido entre 2080-2100, um aumento de temperatura global entre $1,4^{\circ} \mathrm{C}$ e $5,8^{\circ} \mathrm{C}$, relativamente à média de 1961 a 1990. A região mediterrânica devido à sua localização geográfica será uma das mais afectadas pelas características climáticas desta região, que é muito influenciada pelo clima seco e árido do Norte de África. Prevendo-se um aquecimento global das temperaturas, os principais efeitos poderão ser notados nas alterações dos padrões de pluviosidade que serão mais escassos, o que contribuirá para tornar os solos mais secos e contribuir cada vez mais para a escassez de água, nomeadamente na alimentação dos rios do Sul da Península Ibérica caracterizados, sobretudo por serem rios de regime irregular com períodos de estiagem. Por outro lado poderão também ocorrer chuvas muito fortes e cheias em épocas em que normalmente não é habitual. Toda a região mediterrânica tornar-se-á mais seca, o que provocará grandes impactes nas actividades agroflorestais e consequentemente produzirá efeitos económicos graves.

Este artigo descreve sumariamente o trabalho que se encontra em desenvolvimento, no âmbito da tese de doutoramento do programa doutoral em alterações climáticas e políticas de desenvolvimento sustentável, e procura fazer uma observação e análise aos caminhos percorridos ao longo dos últimos anos sobre este tema, bem como encontrar os actores envolvidos, os objectivos e o que se conseguiu alcançar.

Pretende-se estudar a integração desta problemática na investigação, formação de recursos humanos e políticas públicas nas áreas da agricultura, floresta e desenvolvimento rural. Tentando perceber a evolução, continuidade e inovação, nomeadamente, no desenho das políticas propriamente ditas, na disseminação do conhecimento académico e da formação profissional dos recursos humanos ligados ao sector primário e por outro lado, perceber como tem sido o caminho traçado no âmbito do conhecimento científico ao nível das instituições de investigação, desenvolvimento e inovação. O estudo focará investigação desenvolvida no Instituto Nacional de Investigação Agrária e Veterinária, I.P., Laboratório do Estado do Ministério da Agricultura, Florestas e Desenvolvimento Rural.

\section{As Alterações Climáticas na região mediterrânica}

As Alterações Climáticas - AC são identificadas "como uma das maiores ameaças ambientais, sociais e económicas que o planeta e a humanidade enfrentam". Nas últimas décadas tem-se vindo a verificar um aumento considerável de secas, de cheias e outros eventos extremos. A ocorrência

\footnotetext{
${ }^{1}$ O Projeto "Climate Change in Portugal. Scenarios, Impacts and Adaptation Measures" (SIAM) iniciou-se em meados de 1999, com o financiamento da Fundação Calouste Gulbenkian e da Fundação para a Ciência e a Tecnologia.

${ }^{2}$ O projeto "Clima e Meteorologia dos Arquipélagos Atlânticos" (CLIMAAT), foi desenvolvido no âmbito da iniciativa comunitária INTERREG_III B.
} 
destes fenómenos é cada vez mais frequente e a sua intensidade também tem vindo a aumentar. Estes e outros eventos atmosféricos têm vindo a acontecer à escala global e estão a afectar o equilíbrio de alguns ecossistemas e até a sua própria continuidade.

As AC são um tema transversal de estudo e tem suscitado a atenção de cientistas de várias especialidades que tentam procurar soluções de adaptação e mitigação das AC.

Os principais efeitos notados devido às $A C$ são o aquecimento global das temperaturas, alterações dos padrões de pluviosidade (mais escassos), os solos mais secos, a escassez de água, uma insuficiente alimentação dos rios da Península Ibérica. Toda a região mediterrânica tornar-se-á mais seca, o que provocará grandes impactes nas atividades agroflorestais e consequentemente produzirá efeitos económicos graves.

De acordo com diversos estudos, o clima está a mudar globalmente, há regiões do Mundo que serão mais afetadas do que outras devido à sua predisposição natural e localização geográfica. "Devido à variabilidade regional e à gravidade dos impactos climáticos, a maioria das medidas de adaptação deve ser tomada ao nível nacional, regional ou local” (CCE 2009). A região mediterrânica irá ser das mais afetadas no Mundo. As alterações dos padrões dos regimes de pluviosidade bem como a probabilidade de uma maior ocorrência de eventos extremos, como secas severas, ondas de calor, excesso de insolação e ventos com rajadas muito fortes, contribuirão acentuadamente para a problemática da desertificação. Estes eventos levarão à perda de matéria orgânica e portanto a capacidade de retenção de água nos solos diminuirá ou desaparecerá, o que nos sistemas de regadio é fundamental para a competitividade das culturas mediterrânicas, que provavelmente serão extintas se não se criarem medidas e técnicas de adaptação para estas regiões. Estes fatores, além do aumento da temperatura, vão precipitar o acentuar das consequências climáticas naturais em algumas regiões, que potenciarão a desertificação, a poluição e a pobreza, e muitas pessoas ver-seão obrigadas a deslocarem-se para outras regiões do Mundo para conseguirem subsistir.

O conceito de Desenvolvimento Sustentável foi utilizado pela primeira vez no Relatório de Brundtland, elaborado pela Comissão Mundial sobre o Meio Ambiente e o Desenvolvimento e publicado em 1987, com o título "O Nosso Futuro Comum", que define "o desenvolvimento que satisfaz as necessidades presentes, sem comprometer a capacidade das gerações futuras de suprirem as suas próprias necessidades". O desenvolvimento sustentável é um conceito que abrange várias áreas, assentando essencialmente num ponto de equilíbrio entre o crescimento económico, equidade social e a protecção do ambiente. O equilíbrio destes três pilares fundamentais define este conceito. Trata-se de um conceito ainda impreciso e com lacunas e talvez inatingível para a humanidade, porém é utilizado de uma forma simbólica para definir o que se julga ser aceitável em termos de sustentabilidade do Planeta.

Olhando para o problema cada vez mais pertinente que são as $A C$ sob várias perspectivas, sabe-se que os limites de sustentabilidade foram ultrapassados, já não havendo retorno possível. É portanto necessário e urgente adaptar a vida, a economia e o ambiente aos recursos que existem e 
fazer tudo o que estiver ao alcance da humanidade de forma a preservar os recursos naturais possíveis.

\section{1- Efeitos em cascata dos impactos das AC}

Umas das graves consequências dos efeitos das $A C$ é a perda de biodiversidade à escala global (SANTOS, 2012), aspeto crucial para a sustentabilidade do desenvolvimento humano. Os efeitos das AC na atmosfera têm impactos em cascata em vários sectores até chegarem ao nível da segurança alimentar e nutricional (GITZ, 2016). O aquecimento global provoca alterações na composição da atmosfera, que provocam variações na temperatura, na precipitação, que por sua vez vão ter repercussões tais como a acidificação dos oceanos, a degradação dos solos, o aumento do nível do mar e a disponibilidade de água. Todos estes fatores vão contribuir para alterar e degradar os agro-ecossistemas, nomeadamente as espécies aquáticas, as terras cultivadas, a pecuária, as florestas, através do aparecimento de novas pragas e doenças. "Os ecossistemas naturais providenciam à humanidade um vasto e diversificado conjunto de serviços essenciais à vida. Constituem o suporte da biosfera, uma componente do sistema terrestre fundamental para assegurar o seu equilíbrio actual" (SANTOS, 2012, 89). "Além das alterações climáticas e da acidificação dos oceanos, muitas outras alterações ambientais já estão a degradar as terras de cultivo e a ameaçar a produtividade agrícola" (SACHS, 2017, 362). "A agricultura é uma grande fonte de emissões de $\mathrm{CO}_{2}$ através do uso do solo, mas também uma grande fonte dos dois outros principais gases de efeito de estufa" (SACHS, 2017, 367) o metano $\left(\mathrm{CH}_{4}\right)$ emitido por determinadas culturas, nomeadamente do arroz e também pelo gado, e o óxido nitroso $\left(\mathrm{N}_{2} \mathrm{O}\right)$, proveniente de fertilizantes.

Os eventos extremos cada vez mais frequentes, contribuem ainda para intensificar as modificações quer nos ciclos naturais, quer nos diversos ecossistemas. Estes fenómenos produzem efeitos na produção agrícola e pós-colheita, nos mercados e comércio, provocando reflexos económicos e sociais, nomeadamente no consumo e disponibilidade de bens, no aumento de preços e na diminuição da quantidade e da qualidade dos bens de consumo. Os efeitos em cascata traduzem-se por fim em graves problemas de segurança alimentar e nutricional, pois afetam a disponibilidade, o acesso, a utilização e a estabilidade dos bens de consumo alimentar e as suas características nutricionais.

\section{2- As AC na agricultura e desenvolvimento rural como objecto de estudo}

Quando se começou a constatar que os impactes das AC têm consequências em diversas áreas, sendo portanto, um problema transversal e global, a agricultura, os solos, os recursos hídricos e a biodiversidade emergiram como setores de atenção primordial. "Em ciência não existem verdades absolutas, apenas resultados e conclusões que têm maior ou menor probabilidade de descrever e prever os fenómenos com precisão" (SANTOS, 2012, 127). A nível da agricultura, as AC previstas afectarão o rendimento das culturas, a gestão de explorações pecuárias e a localização da produção. 
A probabilidade e a gravidade crescentes dos fenómenos meteorológicos extremos vão ampliar, de forma considerável, o risco de quebra da produção agrícola. (CCE 2009).

Este estudo ${ }^{3}$ pretende fazer a ligação entre as políticas públicas, o ensino superior e formação profissional e a investigação. $O$ trabalho será desenvolvido sob estes três aspectos distintos mas que se interligam, no sentido de encontrar soluções para problemas identificados no âmbito das alterações climáticas. Pretende-se perceber quais são as áreas mais afectadas e mais problemáticas dos dias actuais e que devem merecer uma maior atenção e prioridade no desenho das políticas públicas, nos programas de investigação e na formação dos recursos humanos, em Portugal.

Quando se começou a olhar para a agricultura sob a perspectiva das AC? Para que se estuda as $A C$ na agricultura, para mitigar as consequências ou para adaptar a agricultura às $A C$ ? Quais são os caminhos que têm sido percorridos e para onde se está a caminhar? Quais são os temas mais abordados, ou mais preocupantes nesta área?

Estas são algumas das interrogações que se podem colocar quando se pensa, na alimentação humana, na conservação dos recursos naturais e no desenvolvimento agrícola sustentável. $\mathrm{Na}$ área da investigação em AC como tem sido abordado este assunto pelos organismos da Administração Pública Central, focando sobretudo o desenvolvimento de estudos e projectos no Instituto Nacional de Investigação Agrária e Veterinária, I.P. - INIAV . O INIAV é o Laboratório de Estado, da área de competências da Agricultura, Florestas e Desenvolvimento Rural, que desenvolve atividades de investigação nas áreas agronómica e veterinária e foi criado no âmbito do PREMAC $^{5}$ em 2012, por fusão de vários organismos. Ao longo dos anos, investigadores e técnicos do INIAV têm vindo a realizar estudos e projectos na procura de soluções de desenvolvimento tecnológico e inovação no âmbito das competências do Instituto. Os resultados de muitos destes estudos foram apresentados através de publicações e comunicações. Esta transferência e difusão do conhecimento, a par da formação dos recursos humanos, foram, são e serão um contributo para a melhoria e introdução de técnicas e sistemas de produção mais adaptados às novas realidades emergentes com as mudanças do clima, bem como de ir ao encontro de soluções para minimizar os danos económicos de pragas e doenças nas várias culturas e povoamentos florestais.

\section{3- Abordagem às $A C$ em três vertentes}

Para analisar a problemática identificada, a tese será desenvolvida sob três aspectos distintos mas que se interligam no sentido de procurar resolver problemas identificados no âmbito das alterações climáticas. Assim, os três temas de base serão, as políticas públicas, o ensino académico e formação profissional e a investigação desenvolvida no INIAV relacionada com AC. Serão

\footnotetext{
3 Tese de doutoramento no âmbito do Programa doutoral em alterações climáticas e políticas de desenvolvimento sustentável (UL, UNL e U East Anglia).

${ }^{4} \mathrm{O}$ INIAV foi criado em 2012, no âmbito do PREMAC, ficando com as atribuições relacionadas com a investigação agrária (do L-INIA) e veterinária (do L-LNIV) do antigo Instituto Nacional dos Recursos Biológicos I.P. (INRB), tendo as atribuições deste relativas às áreas das pescas e da aquicultura sido incorporadas no Instituto Português do Mar e da Atmosfera, I. P. (IPMA).

5 PREMAC - Plano de Redução e Melhoria da Administração Central.
} 
elencados os temas mais estudados na agricultura e desenvolvimento rural, nomeadamente novas culturas em determinados locais, novas técnicas que podem ser implementadas devido às alterações de clima, bem como no controlo de doenças e de pragas. De uma forma geral pretende-se perceber como se está a operacionalizar a adaptação da agricultura em virtude das AC e a sua distribuição geográfica no nosso país.

\section{- Políticas Públicas}

"A adaptação tem de ser integrada nas políticas da União Europeia. Este exercício deve ser cuidadosamente preparado, baseando-se numa sólida análise científica e económica. Cada domínio político deveria efectuar uma análise da forma como as políticas poderão ser reorientadas ou alteradas para favorecer a adaptação." (CCE, 2009) No que diz respeito às "políticas públicas agrícolas, florestais e de desenvolvimento rural", será feita uma resenha à legislação portuguesa e comunitária, à $\mathrm{PAC}^{6}$, bem como ao $\mathrm{PDR} 2020^{7}$ e aos $\mathrm{PO}^{8}$ regionais, que permitirá perceber a evolução na formulação das políticas públicas (Tabela I), bem como perceber ao nível da EU, se na implementação das mesmas se conseguem identificar padrões, quer ao nível geográfico, quer ao nível económico.

Tabela I - AC nas políticas públicas de agricultura, floresta e desenvolvimento rural

\begin{tabular}{|l|}
\hline \multicolumn{1}{|c|}{ As AC nas políticas públicas de agricultura, floresta e desenvolvimento rural } \\
\hline União Europeia \\
\hline Política Nacional \\
\hline Política Regional / Planos / Programas \\
\hline Organismos responsáveis pela aplicação das políticas públicas \\
\hline Implementação \\
\hline
\end{tabular}

Fonte: Elaboração própria

Esta análise pretende identificar a evolução das políticas ao longo dos anos no sentido de se verificar a crescente preocupação com a produção agrícola e o desenvolvimento rural, mas também com a preocupação da preservação ambiental, preservação de solos e políticas florestais.

\footnotetext{
${ }^{6}$ PAC - Política Agrícola Comum

${ }^{7}$ Programa de Desenvolvimento Rural 2020

${ }^{8}$ Programas Operacionais
} 


\section{- Formação Profissional e Ensino Superior}

O segundo tema, pretende-se identificar quais são os organismos de ensino superior em Portugal que se têm debruçado sobre a problemática das AC e como tem sido incorporado nos curricula. Por outro lado é igualmente importante saber como tem sido feita a transmissão do conhecimento (Tabela II) para os profissionais, quer de organismos da administração pública, quer de associações agrícolas e florestais e como tem sido a evolução da formação profissional destes recursos ao longo dos anos.

Tal como foi referido em 2009, no Livro Branco da Comissão das Comunidades Europeias Adaptação às alterações climáticas: para um quadro de acção Europeu, "no âmbito da PAC, os Estados-Membros devem inserir a adaptação às alterações climáticas nas três vertentes do desenvolvimento rural vocacionadas para a melhoria da competitividade, o ambiente e a qualidade de vida nas zonas rurais.

Tabela II - Transmissão do conhecimento: AC na formação de Recursos Humanos - formação profissional

\begin{tabular}{|l|}
\hline \multicolumn{1}{|c|}{ Formas de Transmissão do conhecimento } \\
\hline Educação/ Formação \\
\hline Associações de Agricultores \\
\hline Autarquias \\
\hline Universidades \\
\hline Organismos do Estado \\
\hline Entidades de formação e consultoria \\
\hline
\end{tabular}

Fonte: Elaboração própria

Assim como, deve ser analisada a aplicabilidade de medidas a uma escala territorial que transcenda o nível da exploração agrícola. O sistema de aconselhamento agrícola pode ser utilizado para divulgar conhecimentos e incentivar a adopção de novos métodos e tecnologias de gestão das explorações que facilitam a adaptação às alterações climáticas." (CCE 2009)

No que diz respeito ao ensino superior (Tabela III) pretende-se saber como tem sido a evolução do ensino, nomeadamente, na procura de cursos, na inserção desta matéria nos curricula dos cursos já existentes, bem como a criação de novos cursos superiores com matérias relacionadas sobretudo com as AC. A natureza política das AC não é apenas uma barreira, mas também uma oportunidade para investigadores e universidades mostrarem liderança na ação climática (FILHO, et al. 2018). 
Tabela III - Inovação e Desenvolvimento em AC: ensino superior e investigação

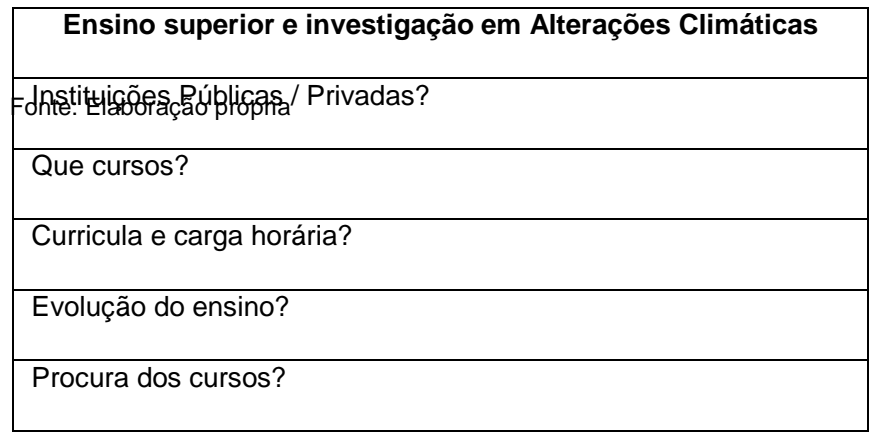

Fonte: Elaboração própria

\section{- Investigação no INIAV}

Relativamente ao terceiro tema, investigação desenvolvida por investigadores do INIAV, o presente estudo pretende analisar e identificar estudos realizados no Instituto ao longo dos anos, que possam de alguma forma contribuir para alargar o conhecimento sobre a adaptação e mitigação das $\mathrm{AC}$, ainda que o objectivo desses estudos não tenha sido esse. Além das pesquisas pretende-se realizar algumas entrevistas com investigadores do INIAV que tenham participado nestes estudos. Os resultados destes projectos de investigação levaram ao desenvolvimento de técnicas de diagnóstico e combate de pragas e doenças de determinadas culturas e povoamentos florestais, à percepção das melhores condições climáticas para a produção de determinadas culturas e também de técnicas utilizadas que possam contribuir para a conservação dos solos e uso mais eficiente da água, assim como para a conservação e valorização dos recursos genéticos regionais. Uma análise dos objectivos e resultados de estudos e projectos selecionados permitirá fazer uma compilação de técnicas e conclusões que possam contribuir para a apdatação e mitigação das AC.

\section{4- Metodologia adotada}

\section{- Instrumentos de recolha de informação}

A aplicação de uma estratégia metodológica engloba várias técnicas de análise, nomeadamente quantitativa e qualitativa. A principal fonte de informação será secundária (relatórios institucionais, estatísticas oficiais, legislação, curricula escolares), mas também será feita a recolha de informação primária através de vários métodos, a saber, um inquérito por questionário, entrevistas e a formação de grupos focais. Quanto à forma de construção destes instrumentos, para a realização das respectivas análises quantitativa e qualitativa, será aplicado primeiro o inquérito por questionário. Posteriormente serão realizadas entrevistas compreensivas e grupos focais, a uma amostra a definir com os dados recolhidos através do inquérito por questionário. 
Da recolha deste material, será realizada uma análise documental ao conteúdo dos documentos. «A análise documental faz-se principalmente, por classificação-indexação; a análise categorial temática é, entre outras, uma das técnicas da análise de conteúdo» (Bardin, 2007:41). O objectivo da análise documental, é a representação condensada da informação, que vai evidenciar indicadores que irão permitir inferir sobre uma realidade que não a da mensagem. Esta técnica consiste num conjunto de operações, que contribuem para a elaboração de um novo documento diferente do original que irão facilitar a sua consulta posterior mas também separar a informação através da categorização. A classificação pressupõe várias classes definidas pelo investigador, segundo alguns critérios comuns encontrados nos documentos, nomeadamente a utilização de expressões ou palavras, que possam levar a uma melhor compreensão bem como inferir, ou seja, extrair elementos subjacentes nos documentos, que não são perceptíveis numa simples leitura, e que poderão ser melhor compreendidos, num conjunto de diferentes documentos. Desta análise é expectável que se consiga encontrar algumas respostas que contribuam para um maior esclarecimento do objecto de estudo.

\section{- Análise de conteúdo}

A análise de conteúdo nesta investigação vai realizar-se em dois momentos distintos. O primeiro aplicando esta técnica às respostas dadas, às questões de resposta aberta, por todos os respondentes ao inquérito por questionário, e o segundo momento às respostas dadas nas entrevistas. "A análise de conteúdo é um conjunto de técnicas de análise das comunicações (...) marcado por uma grande disparidade de formas e adaptável a um campo de aplicação muito vasto» (Bardin, 2007:27). Para aplicar esta técnica é necessário recorrer à classificação por categorias que podem ser definidas à partida pelo investigador, ou podem surgir no momento da análise dos documentos. Esta categorização vai permitir reduzir a complexidade da informação. As categorias devem ser bem formuladas e adaptáveis ao objecto em análise. A análise de conteúdo vai permitir reduzir substancialmente a dimensão dos documentos, e permitir através da análise posterior, que utiliza o número de frequências e de ocorrências das categorias, uma melhor interpretação e a construção de tipologias. A construção de novos dados a partir desta técnica vai traduzir-se numa melhor interpretação dos resultados, e observar semelhanças ou diferenças que de outra forma não se conseguiria retirar duma análise da leitura dos documentos.

\section{- Entrevistas}

As entrevistas a realizar serão do tipo compreensivo "a aplicação da entrevista de tipo compreensivo pressupõe a obtenção de um discurso mais narrativo que informativo, resultado da intersubjectividade que se desenrola entre entrevistado e entrevistador" (Ferreira, 2014). Numa abordagem qualitativa, o que se procura não é uma representatividade estatística, mas sim uma "representatividade social", como refere Guerra. O objectivo do investigador não é demonstrar mas compreender. Tem como base uma hipótese bem consolidada e procura-se confirmar ou infirmar 
numa lógica mais compreensiva. Procura-se compreender comportamentos sem ter como pressuposto um quadro de hipóteses muito bem definidas. "As análises compreensivas têm em comum a assunção da concepção weberiana do sujeito, que o considera capaz de ter racionalidades próprias e comportamentos estratégicos que dão sentido às suas opções num contexto sempre em mudança provocada pela sua própria acção" (Guerra, 2006:17). Numa entrevista compreensiva, o entrevistado é o informador privilegiado, e não um informador objectivo como numa pesquisa do tipo mais cartesiano (Guerra, 2006). Integra-se o objecto de estudo com a entrevista compreensiva.

\section{Considerações}

Sendo as alterações climáticas uma das maiores ameaças a nível global é urgente a tomada de medidas capazes de minimizar os seus impactes. A partir do que foi explanado, pretende-se chegar a resultados que permitam encontrar novas pistas que venham a contribuir de uma forma positiva e enriquecedora para mitigar e adaptar as consequências das alterações climáticas.

Ao nível da agricultura e do desenvolvimento rural é necessário conhecer quais são os problemas mais sentidos e as necessidades reais dos agricultores e produtores, para se poder agir em conformidade. Uma análise à evolução das políticas públicas, sobretudo da PAC, bem como, às opções políticas tomadas pelos vários Governos Constitucionais, poderá permitir identificar lacunas, nomeadamente na transmissão de conhecimentos especializados a quem está no terreno.

A capacitação de recursos humanos, em novas áreas de formação profissional, possibilitará ir ao encontro de novas formas de actuação ao nível local contribuindo para a minimização deste problema global.

\section{Bibliografia}

BARDIN, Laurence (2007) Análise de Conteúdo, Lisboa: Edições 70. [ed. original: 1977]

FERREIRA, Vitor Sérgio (2014) Artes e manhas da entrevista compreensiva.

http://www.scielo.br/scielo.php?script=sci_arttext\&pid=S0104-12902014000300979\&lng=pt\&tlng=pt

FILHO, W. L. et al. (2018). Implementing climate change research at universities: Barriers, potential actions. Journal of Cleaner Production, 170 (2018), 269-277.

GIDDENS, Anthony (2010), A política da mudança climática. Rio de Janeiro. Zahar

GITZ, V. et al (2016). Climate change and food security: risks and responses. Mediterranean Agriculture and Climate Change. Impacts, adaptations, solutions. Paris: CIHEAM, Watch Letter n37

GUERRA, Isabel (2006) Pesquisa Qualitativa e Análise de Conteúdo - Sentidos e formas de uso, Estoril, Princípia.

Livro Branco - Adaptação às alterações climáticas: para um quadro de acção europeu - 
$\operatorname{COM}(2009) 147$ final

MORGAN, David L., KRUEGER, Richard A. (1998), The focus group kit - The focus group guidebook, Thousand Oaks, Sage

SACHS. J.D; (2017), A era do desenvolvimento sustentável. Conjuntura Actual Editora.

SANTOS, Filipe Duarte; MIRANDA, Pedro (2006), Alterações Climáticas em Portugal. Cenários, Impactos e Medidas de Adaptação. Lisboa. Gradiva

SANTOS, Filipe Duarte (2012), Alterações Globais: Os desafios e os riscos presentes e futuros. Lisboa. Fundação Francisco Manuel dos Santos

SANTOS, Filipe Duarte (2007), Que Futuro? Ciência, Tecnologia, Desenvolvimento e Ambiente. Lisboa. Gradiva 